\title{
Glutamatergic and Nonglutamatergic Neurons of the Ventral Tegmental Area Establish Local Synaptic Contacts with Dopaminergic and Nondopaminergic Neurons
}

\author{
Alice Dobi, ${ }^{1}$ Elyssa B. Margolis, ${ }^{2}$ Hui-Ling Wang, ${ }^{1}$ Brandon K. Harvey, ${ }^{1}$ and Marisela Morales ${ }^{1}$ \\ ${ }^{1}$ National Institute on Drug Abuse, Intramural Research Program, Baltimore, Maryland 21224, and ${ }^{2}$ Ernest Gallo Clinic and Research Center, University of \\ California, San Francisco, Emeryville, California 94608
}

\begin{abstract}
The ventral tegmental area (VTA) contributes to reward and motivation signaling. In addition to the well established populations of dopamine (DA) or GABA VTA neurons, glutamatergic neurons were recently discovered in the VTA. These glutamatergic neurons express the vesicular glutamate transporter 2, VGluT2. To investigate whether VTA glutamatergic neurons establish local synapses, we tagged axon terminals from resident VTA neurons by intra-VTA injection of Phaseolus vulgaris leucoagglutinin (PHA-L) or an adenoassociated virus encoding wheat germ agglutinin (WGA) and by immunoelectron microscopy determined the presence of VGluT2 in PHA-L- or WGA-positive terminals. We found that PHA-L- or WGA-positive terminals from tagged VTA cells made asymmetric or symmetric synapses within the VTA. VGluT2 immunoreactivity was detected in the vast majority of PHA-L- or WGA-positive terminals forming asymmetric synapses. These results indicate that both VTA glutamatergic and nonglutamatergic (likely GABAergic) neurons establish local synapses. To examine the possible DAergic nature of postsynaptic targets of VTA glutamatergic neurons, we did triple immunolabeling with antibodies against VGluT2, tyrosine hydroxylase (TH), and PHA-L. From triple-labeled tissue, we found that double-labeled PHA-L (+)/VGluT2 (+) axon terminals formed synaptic contacts on dendrites of both TH-positive and TH-negative cells. Consistent with these anatomical observations, in whole-cell slice recordings of VTA neurons we observed that blocking action potential activity significantly decreased the frequency of synaptic glutamatergic events in DAergic and non-DAergic neurons. These observations indicate that resident VTA glutamatergic neurons are likely to affect both DAergic and non-DAergic neurotransmission arising from the VTA.
\end{abstract}

\section{Introduction}

The ventral tegmental area (VTA) plays a role in goal-directed behavior and the reward processing of natural reinforcers and several drugs of abuse (Schultz, 2002; Wise, 2004). It is well established that DAergic and GABAergic neurons are present in the VTA; however, recent observations indicate that neurons with glutamatergic phenotype lacking either DAergic (Kawano et al., 2006; Yamaguchi et al., 2007; Nair-Roberts et al., 2008) or GABAergic (Yamaguchi et al., 2007) phenotypes are also present in the VTA.

Glutamatergic neurons can be identified through the detection of mRNA encoding vesicular glutamate transporters (VGluTs), which transport glutamate into synaptic vesicles at presynaptic terminals. Three isoforms of the vesicular glutamate transporter, VGluT1, VGluT2, and VGluT3, are present in the CNS (Bellocchio

Received Aug. 8, 2009; revised Sept. 2, 2009; accepted Sept. 10, 2009.

This work was supported by the Intramural Research Program of the National Institute on Drug Abuse. E.B.M. was supported by funds from the State of California for medical research on alcohol and substance abuse through the University of California, San Francisco. We thank Doug Howard for assistance with viral vector preparation.

Correspondence should be addressed to Marisela Morales, National Institute on Drug Abuse, Intramural Research Program, Biomedical Research Center, 251 Bayview Boulevard, Baltimore, MD21224.E-mail:mmorales@intra.nida. nih.gov.

A. Dobi's present address: National Institute on Alcohol Abuse and Alcoholism, 5625 Fishers Lane, MSC 9411, Bethesda, MD 20852.

DOI:10.1523/JNEUROSCI.3884-09.2010

Copyright $\odot 2010$ the authors $\quad 0270-6474 / 10 / 300218-12 \$ 15.00 / 0$ et al., 1998; Bai et al., 2001; Fremeau et al., 2001; Fujiyama et al., 2001; Hayashi et al., 2001; Herzog et al., 2001; Takamori et al., 2001; Varoqui et al., 2002). Within the VTA, glutamatergic cells express transcripts encoding VGluT2, but not VGluT1 or VGluT3 (Kawano et al., 2006; Yamaguchi et al., 2007).

DAergic and GABAergic VTA neurons communicate locally within the VTA in addition to their projections to other brain structures. DA modulates local neural activity within the VTA through somatodendritic release of DA that can activate postsynaptic $\mathrm{D}_{2}$ receptors (Johnson and North, 1992b) or presynaptic $\mathrm{D}_{1}$ receptors on GABAergic terminals (Cameron and Williams, 1993). Johnson and North (1992a) provided evidence for local GABAergic connections in the VTA by showing that the frequency of spontaneous inhibitory synaptic events on putative DAergic neurons was decreased by terminating action potential activity with the $\mathrm{Na}^{+}$channel blocker tetrodotoxin (TTX). Such local signaling is likely to regulate the DAergic and non-DAergic neurotransmission to VTA target structures (Swanson, 1982; Fallon et al., 1984; Van Bockstaele and Pickel, 1995; Carr and Sesack, 2000; Margolis et al., 2006).

Because both DAergic and GABAergic neurons of the VTA signal locally, we raised the following question: do VTA glutamatergic neurons also have both intrinsic and extrinsic connections? While some evidence exists suggesting that VTA glutamatergic neurons project to the prefrontal cortex (PFC) (Hur and Zaborszky, 2005; Lavin et al., 2005), or to the nucleus accumbens (Chuhma et 
al., 2004), it is not clear whether or not VTA glutamatergic neurons make local synapses. In the present study we used anatomical and electrophysiological approaches to investigate the local circuitry of VTA glutamatergic neurons. We tagged axon terminals from resident VTA neurons by intra-VTA injection of either the anterograde tracer Phaseolus vulgaris leucoagglutinin (PHA-L) or an adeno-associated virus (AAV) vector expressing cDNA for the tracer wheat germ agglutinin (WGA), and by double immunolabeling and electron microscopy determined whether local PHA-L- or WGA-positive axon terminals contain or lack VGluT2. We also used triple immunolabeling to determine whether VTA glutamatergic neurons form synapses with DAergic neurons. Finally, we used in vitro electrophysiology to test for functional local glutamatergic synapses from intrinsic VTA neurons in acute brain slices. These experiments all provide evidence for glutamatergic synaptic transmission by local glutamatergic neurons in the VTA.

\section{Materials and Methods}

Animals and surgical procedures. For anatomical studies, 66 male Sprague Dawley rats (250-420 g body weight) were anesthetized with an intraperitoneal injection of $3 \mathrm{ml} / \mathrm{kg}$ chloral hydrate $(40 \mathrm{mg} / \mathrm{ml})$ in physiological saline. Anesthetized rats were fixed in a stereotaxic apparatus and either the anterograde axonal tracer PHA-L $(2.5 \%$ in $0.01 \mathrm{M}$ sodium phosphate buffer, pH 7.8; Vector Laboratories) or AAV-WGAtr (see below, Vector construction and packaging) was delivered into the VTA (bregma -5.2, ML +0.8, DV - 8.4). PHA-L was applied iontophoretically into the VTA of 50 rats through a stereotaxically positioned glass micropipette (inner tip diameter $10-15 \mu \mathrm{m}$ ) by applying $5 \mu \mathrm{A}$ current, $5 \mathrm{~s}$ on/off for $15 \mathrm{~min}$. A second group of 16 rats was injected with AAVWGAtr $\left(25 \mathrm{nl}\right.$ of $4 \times 10^{12}$ viral genomes $/ \mathrm{ml} 0.01 \mathrm{M} \mathrm{PB}$ containing $0.5 \mathrm{~mm}$ magnesium chloride, $\mathrm{pH}$ 7.4) by pressure through a glass micropipette attached to a Picospritzer III (Parker Hannifin). Seven days after injection of PHA-L or 3 weeks after injection of AAV-WGAtr, the rats were deeply anesthetized with chloral hydrate $(35 \mathrm{mg} / 100 \mathrm{~g})$ and perfused transcardially with either $500 \mathrm{ml}$ of fixative containing $4 \%(\mathrm{~W} / \mathrm{V})$ paraformaldehyde, $0.05 \%$ glutaraldehyde and $15 \%$ picric acid in $0.1 \mathrm{M}$ phosphate buffer ( $\mathrm{PB}, \mathrm{pH} 7.3$ ) or with $75 \mathrm{ml}$ of fixative containing $3.75 \%$ acrolein and $2 \%$ paraformaldehyde followed with $300 \mathrm{ml}$ of $2 \%$ paraformaldehyde. Brains were removed and kept in $2 \%$ paraformaldehyde for $2 \mathrm{~h}$. The midbrains were cut into coronal serial sections (50 $\mu \mathrm{m}$ thick) with a vibratome (VT1000S, Leica). The detection of antigens was similar when we compared brain sections of animals perfused with either of the two fixatives. However, the best ultrastructural preservation was obtained with the fixative solution containing acrolein. All animal procedures were approved by the National Institute on Drug Abuse Animal Care and Use Committee.

Vector construction and packaging. Using RT-PCR, the cDNA for wheat (Triticum aestivum) germ agglutinin (kindly provided by Dr. Yoshihara, RIKEN Brain Science Institute, Tokyo, Japan) was truncated after codon 198 (Ala) and flanked by KpnI sites. The resulting PCR fragment was digested with KpnI and inserted into the KpnI site of pAAV-GFP (Xiao et al., 1998). The resulting plasmid, pAAV-WGAtr, was sequence verified and used to prepare viral stocks by the triple-transfection method (Xiao et al., 1998; Howard et al., 2008). Briefly, $2015 \mathrm{~cm}$ dishes containing HEK293 cells at $85-95 \%$ confluency were transfected by $\mathrm{CaCl}_{2}$ method with pHelper (Stratagene), pAAV-WGAtr, and a plasmid containing rep/ cap genes for serotype 9, pAAV9 (Gao et al., 2004). Plasmids used for packaging AAV were generously provided by Dr. Xiao Xiao (UNC, Chapel Hill, NC). Approximately $48 \mathrm{~h}$ post-transfection, cells were harvested, lysed by freeze and thaw, and purified by centrifugation on $\mathrm{CsCl}$ gradient. Final samples were dialyzed in PBS, aliquoted, and stored at $-80^{\circ} \mathrm{C}$ until use. All vectors were titered by quantitative PCR using the CMV promoter as the target sequence. Viral titers were $\sim 4 \times 10^{12}$ viral genomes/ml.

Immunolabeling for light microscopy. To determine whether the injected PHA-L or transduced WGA was confined to VTA neurons, every sixth section of the VTA was used to detect PHA-L or WGA by immu- nohistochemistry. Sections were rinsed with PBS, pH 7.4, and incubated with a blocking solution [10\% normal goat serum (NGS) in PBS supplemented with $0.3 \%$ Triton X-100] for $1 \mathrm{~h}$. Sections were then incubated with either rabbit anti-PHA-L antibody (1:2000, Vector Laboratories), rabbit anti-WGA (T 4144; Sigma, 1: 20,000) or goat anti-WGA antibody (AS-2024; Vector Laboratories, 1:500 dilution) in the blocking solution overnight at $4^{\circ} \mathrm{C}$. After rinsing $3 \times 10 \mathrm{~min}$ in PBS, sections were processed with an ABC kit (Vector Laboratories). The sections were incubated for $1 \mathrm{~h}$ at room temperature with a 1:200 dilution of the biotinylated anti-rabbit or anti-goat secondary antibody, rinsed with PBS, and incubated with avidin-biotinylated horseradish peroxidase for $1 \mathrm{~h}$. Sections were rinsed and the peroxidase reaction was then developed with $0.05 \% 3$, 3-diaminobenzidine- $4 \mathrm{HCl}(\mathrm{DAB})$ and $0.003 \%$ hydrogen peroxide $\left(\mathrm{H}_{2} \mathrm{O}_{2}\right)$. Following evaluation of PHA-L or WGA immunoreactivity in VTA cells, brain sections of rats with PHA-L- or WGA-immunoreactivity within the parabrachial pigmental area (PBP) or paranigral nucleus (PN) of the VTA were processed for immunoelectron microscopy. The specificity of primary anti-PHA-L antibodies was demonstrated by the lack of PHA-L immunolabeling in brain sections from rats injected with saline solution without PHA-L.

Double immunolabeling for fluorescent microscopy. Coronal brain sections $(50 \mu \mathrm{m})$ of AAV-WGA-injected rats were incubated for $1 \mathrm{~h}$ in $\mathrm{PB}$ supplemented with $4 \%$ BSA and $0.3 \%$ Triton X-100. Sections were then incubated with a mixture of goat anti-WGA antibody (AS-2024; Vector Laboratories, 1:500 dilution) and mouse anti-TH antibody (MAB318; Millipore Bioscience Research Reagents, 1:500 dilution) overnight at $4^{\circ} \mathrm{C}$. After being rinsed $3 \times 10 \mathrm{~min}$ in $\mathrm{PB}$, sections were incubated in a mixture of Texas Red donkey anti-mouse (715-075-150; Jackson Immunoresearch Laboratories, 1;100 dilution) and FITC donkey anti-goat (705095-147; Jackson Immunoresearch Laboratories, 1:100 dilution). After being rinsed, sections were mounted on slides and air dried.

Immunolabeling for electron microscopy. Vibratome tissue sections were rinsed with $50 \mathrm{~mm}$ Tris-buffered saline (TBS, pH 7.4), incubated with $1 \%$ sodium borohydride in TBS for $30 \mathrm{~min}$ to inactivate free aldehyde groups, rinsed in TBS, and then incubated with $0.05 \%$ Triton-X for $50 \mathrm{~min}$ followed by blocking in TBS containing 20\% NGS for $30 \mathrm{~min}$. Sections were then incubated with primary antibodies as follows: rabbit anti-PHA-L antibody (single labeling), rabbit anti-WGA (single labeling), rabbit anti-PHA-L plus guinea pig anti-VGluT2 antibodies (double labeling), rabbit anti-WGA plus guinea pig anti-VGluT2 antibodies (double labeling), rabbit anti-PHA-L plus guinea pig anti-VGluT1 antibodies (double labeling), or rabbit anti-PHA-L plus guinea pig antiVGluT2 plus mouse anti-TH antibodies (triple labeling). All primary antibodies were diluted in TBS with $2 \%$ NGS, and incubations were for $48 \mathrm{~h}$ at $4^{\circ} \mathrm{C}$. The primary antibodies were used at 1:2000 dilution for rabbit anti-PHA-L antibody (Vector Laboratories), 1:20,000 dilution for rabbit anti-WGA, 1:8000 for guinea pig anti-VGluT2 antibody (AB5907; Millipore Bioscience Research Reagents), 1:5000 for guinea pig antiVGluT1 antibody (AB5905; Millipore Bioscience Research Reagents), and 1:2000 for mouse anti-TH antibody (MAB318; Millipore Bioscience Research Reagents). Sections were rinsed and incubated overnight at $4^{\circ} \mathrm{C}$ in the corresponding secondary antibodies: biotinylated goat anti-rabbit antibodies (1:100, Vector Laboratories) for single labeling or in a mixture of secondary antibodies containing biotinylated goat anti-rabbit antibodies and mouse monoclonal anti-guinea pig IgG (1:1000; G0522; Sigma-Aldrich) against VGluT2 primary antibody or a mixture of secondary antibodies containing biotinylated goat anti-rabbit antibodies and anti-guinea pig IgG (Fab' fragment, 1:100 dilution) coupled to 1.4 $\mathrm{nm}$ gold (Nanoprobes) against VGluT1 primary antibody in TBS with $2 \%$ NGS. After several rinses, sections for VGluT2 and TH labeling were incubated with anti-mouse IgG (Fab' fragment, 1:100 dilution) coupled to $1.4 \mathrm{~nm}$ gold (Nanoprobes) overnight at $4^{\circ} \mathrm{C}$. Sections for VGluT1, VGluT2, and TH labeling were rinsed in TBS and then in double-distilled water, followed by silver enhancement of the gold particles with the Nanoprobe Silver Kit (Nanoprobes) for $7 \mathrm{~min}$. Next, all sections were incubated in avidin-biotinylated horseradish peroxidase complex (ABC, 1: 100 dilution; Vector Laboratories) in TBS for $2 \mathrm{~h}$ at room temperature and washed in Tris-buffer (TB, pH 7.4). Peroxidase activity was detected by placing the sections in a solution containing $\mathrm{DAB}(0.025 \%)$ and 
$0.003 \% \mathrm{H}_{2} \mathrm{O}_{2}$ in $\mathrm{PB}$ for 3 min. Peroxidase reaction was stopped by rinsing sections in PBS, sections were fixed with $2 \%$ osmium tetroxide in PBS for 25 min, washed in PBS followed by double-distilled water and then contrasted in freshly prepared $1 \%$ uranyl acetate for $35 \mathrm{~min}$. Sections were dehydrated through a series of graded alcohols and with propylene oxide. Afterward, they were flat embedded in Durcupan ACM epoxy resin (Electron Microscopy Sciences). Resin-embedded sections were polymerized at $60^{\circ} \mathrm{C}$ for $2 \mathrm{~d}$. Sections of $70 \mathrm{~nm}$ were cut from the outer surface of the tissue with an ultramicrotome (Leica Microsystems) using a diamond knife (Diatome). The sections were collected on Formvar-coated single slot grids and counterstained with Reynolds lead citrate (Reynolds, 1963). Sections were examined and photographed using a Tecnai $\mathrm{G}^{2} 12$ transmission electron microscope (Fei) equipped with a digital micrograph 3.4 camera (Gatan).

Specificity of primary anti-VGluT2 and anti-VGluT1 antibodies were demonstrated by results showing that preincubation of the primary antibodies with the antigenic peptide abolished positive VGluT2 and VGluT1 immunolabeling, as we previously described (Tagliaferro and Morales, 2008). We showed that the anti-TH antibody labels a single protein band in the molecular weight range of $56-60 \mathrm{kDa}$ on Western blots prepared from VTA protein homogenates (Shepard et al., 2006). In addition, we had used this anti-TH antibody to demonstrate that midbrain unilateral lesions with 6-OHDA results in lack of TH immunolabeling in the lesioned site, while is preserved in the contralateral unlesioned midbrain (Sarabi et al., 2001). Omitting one of the primary antibodies during double or triple labeling removed corresponding labeling and confirmed no cross-reaction between different primary antibodies.

Ultrastructural analysis. Coronal vibratome sections of VTA corresponding to plates $71-79$ (bregma $-4.56 \mathrm{~mm}$ to $-5.52 \mathrm{~mm}$ ) of the rat brain atlas of Paxinos and Watson (2007) were used in this study. Four to six grids containing thin sections were collected from four to six plasticembedded sections of the VTA from a total of six rats: three PHA-L- and three WGA-injected rats. Synaptic contacts were classified according to their morphology and immunolabel, and photographed at a magnification of $6800-13,000 \times$. The morphological criteria used for identification and classification of cellular components observed in these thin sections were as previously described (Peters et al., 1991). Briefly, neuronal cell bodies were identified by the presence of a large and round nucleus as well as rough endoplasmic reticulum and Golgi apparatus. Large and medium sized dendrites were identified by the presence of endoplasmic reticulum, mitochondria and microtubules. Dendritic spines were identified by the absence of mitochondria. Axon terminals were characterized by the presence of synaptic vesicles and mitochondria, whereas axon preterminals and unmyelinated axons are smaller in diameter and occasionally contain a small number of synaptic vesicles. Type 1 synapses, referred to here as asymmetric synapses, were characterized by the presence of thick postsynaptic densities, whereas type 2 synapses are referred to as symmetric synapses and have thin postsynaptic densities. When the identification of a synaptic contact was not clear, contiguous sections were obtained to determine the type of synapse. Pictures were adjusted to match contrast and brightness by using Adobe Photoshop (Adobe Systems).

Slice preparation and electrophysiology. Male Sprague Dawley rats (33-40 d old) were anesthetized with isofluorane, and their brains were removed. Horizontal slices ( $250 \mu \mathrm{m}$ thick) containing the VTA were prepared using a vibratome (Leica Instruments, Germany). Slices were submerged in artificial CSF (ACSF) containing (in mM) $119 \mathrm{NaCl}$, $2.5 \mathrm{KCl}, 1.3 \mathrm{MgSO}_{4}, 1.0 \mathrm{NaH}_{2} \mathrm{PO}_{4}, 2.5 \mathrm{CaCl}_{2}, 26.2 \mathrm{NaHCO}_{3}$, and 11 glucose saturated with $95 \% \mathrm{O}_{2}-5 \% \mathrm{CO}_{2}$ and allowed to recover at $34^{\circ} \mathrm{C}$

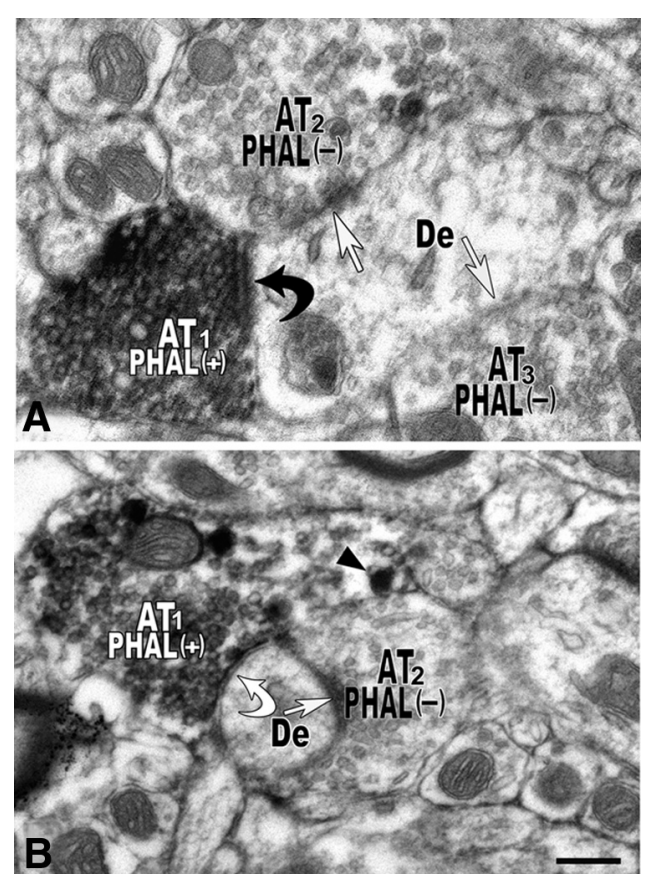

Figure 2. Electron micrographs of PHA-L-labeled axon terminals forming asymmetric or symmetric synapses on dendrites in the VTA. $\boldsymbol{A}$, Example of an axon terminal $\left(\mathrm{AT}_{1}\right)$ containing PHA-L immunolabeling [PHAL $(+)]$ and forming an asymmetric synapse (black curved arrow) with a dendrite (De) receiving synapses (white straight arrows) from two unlabeled axon terminals $\left(\mathrm{AT}_{2}\right.$ and $\left.A \mathrm{~T}_{3}\right) . B$, Axon terminal $\left(\mathrm{AT}_{1}\right)$ containing PHA-L immunolabeling and large dense core vesicles covered by HRP reaction product (arrowhead) forming a symmetric synapse (white curved arrow) with a dendrite that receives an additional symmetric synapse (white straight arrows) from an unlabeled axon terminals ( $\left(\mathrm{AT}_{2}\right.$ ). Scale bar (in $\boldsymbol{B}$ ): $0.2 \mu \mathrm{m}$ for $\boldsymbol{A} ; 0.26$ $\mu \mathrm{m}$ for $\boldsymbol{B}$.

for at least $1 \mathrm{~h}$. Tissue anterior to the VTA was removed from slices to sever any inputs arising from the hypothalamus (supplemental Fig. 2, available at www.jneurosci.org as supplemental material). Individual slices were visualized under a Zeiss Axioskop with differential interference contrast optics and infrared illumination. Whole-cell patch- 
Table 1. Neurochemical composition of PHA-L-labeled axon terminals and their targets in the rat VTA

\begin{tabular}{|c|c|c|c|c|}
\hline & \multicolumn{2}{|c|}{$\begin{array}{l}\text { PHA-L-labeled terminals synapsing } \\
\text { on dendrites }\end{array}$} & \multicolumn{2}{|c|}{$\begin{array}{l}\text { PHA-L-labeled terminals synapsing } \\
\text { on dendritic spines }\end{array}$} \\
\hline & Asymmetric synapses & Symmetric synapses & Asymmetric synapses & Symmetric synapses \\
\hline PHA-L-labeled terminals with VGluT2 signal $[56 \%(n=30)]$ & $80 \%(n=24)$ & $10 \%(n=3)$ & $10 \%(n=3)$ & $0 \%(n=0)$ \\
\hline PHA-L-labeled terminals without VGluT2 signal [44\% $(n=24)]$ & $13 \%(n=3)$ & $79 \%(n=19)$ & $8 \%(n=2)$ & $0 \%(n=0)$ \\
\hline
\end{tabular}

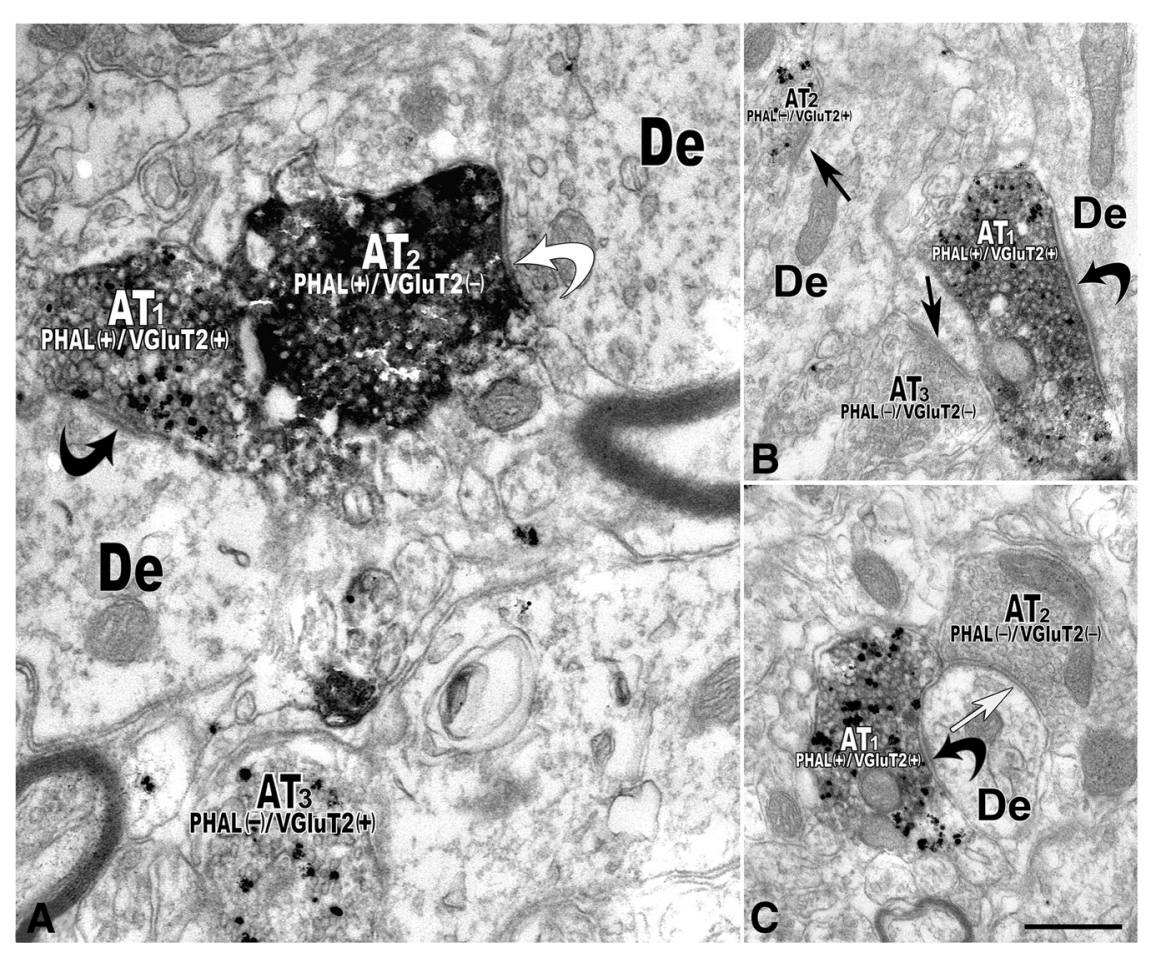

Figure 3. Electron micrographs reveal PHA-L-labeled axon terminals that contain VGluT2 immunolabeling establishing asymmetric synapses on dendrites of local VTA neurons (double immunoelectron microscopy). $A$, Axon terminal ( $\mathrm{AT}_{1}$ ) containing PHA-L (immunoperoxidase product) and VGluT2 (immunogold-silver particles). PHAL(+)/VGluT2(+) axon terminal makes an asymmetric synapse (black curved arrow) with a dendrite (De). The axon terminal ( $\mathrm{AT}_{2}$ ) containing PHA-L but lacking VGluT2 labeling $[\operatorname{PHAL}(+) /$ VGluT2( $(-)]$ forms a symmetric synapse (white curved arrow) with a dendrite (De). Note a third axon terminal (AT $\left.{ }_{3}\right)$ lacking PHA-L but containing VGluT2 [PHAL( $(-) /$ VGluT2 $(+)]$. B, A PHA $(+) /$ VGluT2 $(+)$ axon terminal ( AT $\left._{1}\right)$ makes an asymmetric synapse (curved black arrow) with a dendrite (De). A VGluT2-positive terminal $\left(\mathrm{AT}_{2}\right)$ lacking PHA-L [PHAL(-)/VGluT2(+)] makes an asymmetric synapse (black straight arrow) with a dendrite. An axon terminal $\left(\mathrm{AT}_{3}\right)$ lacking both PHA-L and VGluT2 [PHAL(-)/VGluT2(-)] makes an asymmetric synapse (black straight arrow) with a dendrite. C, PHA-L(+) and VGluT2(+) axon terminal ( $\mathrm{AT}_{1}$ ) establishes an asymmetric synapse (black curved arrow) with a dendrite (De) receiving a PHA-L and VGluT2immunonegative [PHAL( - )/VGluT2(-)] axon terminal $\left(\mathrm{AT}_{2}\right)$ forming a symmetric synapse (white straight arrow). Scale bar (in C): $0.5 \mu \mathrm{m}$ for $A ; 0.4 \mu \mathrm{m}$ for $\boldsymbol{B}$ and $C$.

clamp recordings were made at $36^{\circ} \mathrm{C}$ using $2.5-5 \mu \mathrm{m}$ pipettes containing (in mM) $123 \mathrm{~K}$-gluconate, $10 \mathrm{HEPES}, 0.2 \mathrm{EGTA}, 8 \mathrm{NaCl}, 2$ MgATP, and $0.3 \mathrm{Na}_{3} \mathrm{GTP}$ (pH 7.2, osmolarity adjusted to 275). Biocytin $(0.1 \%)$ was also included in the internal solution to identify DAergic neurons with immunocytochemistry following recordings.

Recordings were made using an Axopatch 1-D (Molecular Devices), filtered at $2 \mathrm{kHz}$ and collected at $5 \mathrm{kHz}$ using procedures written for IGOR Pro (Wavemetrics). All recordings were made in voltage-clamp holding the cells at $V_{\mathrm{m}}=-60 \mathrm{mV}$. $\mathrm{I}_{\mathrm{h}}$ was recorded at the beginning of each experiment by stepping from -60 to $-40,-50,-70,-80,-90,-100,-110$, and $-120 \mathrm{mV}$. Series resistance and input resistance were sampled throughout experiments with $4 \mathrm{mV}, 200$ ms hyperpolarizing steps applied once every $10 \mathrm{~s}$. All excitatory events were measured in the presence of the $\mathrm{GABA}_{\mathrm{A}}$ receptor antagonist picrotoxin $(100 \mu \mathrm{M})$ to isolate glutamatergic events. Events were identified and quantified using procedures developed for IGOR Pro (Wavemetrics). Cells with baseline event frequencies of $<0.25 \mathrm{~Hz}$ were excluded from this study. In a subset of experiments, the external $\mathrm{KCl}$ concentration was raised to $5.0 \mathrm{~mm}$ to depolarize the slice, therefore increasing the baseline event frequency to better measure the effects of the $\mathrm{Na}^{+}$ channel blocker TTX on event frequency. In some experiments the AMPA receptor (AMPAR) antagonist 6,7-dinitroquinoxaline-2,3(1H-4H)dione (DNQX) $(10 \mu \mathrm{M})$ was added after the TTX application to confirm that the measured events were glutamatergic.

Electrophysiological results are presented as means $+/-$ SEM. Summary comparisons of event frequencies and amplitudes were made between the average over the 4 min of baseline just preceding TTX application to the last 4 min of TTX application using a paired Student's $t$ test. All drugs were applied by bath perfusion. Stock solutions were made and diluted in ACSF immediately before application. For stock solutions, TTX was diluted in $\mathrm{H}_{2} \mathrm{O}(1$ $\mathrm{mM})$; picrotoxin (100 $\mathrm{mm}$ ) and DNQX (10 $\mathrm{mM}$ ) were diluted in DMSO. All chemicals were obtained from Sigma Chemical or Tocris Bioscience.

Immunocytochemistry for recording slices. Immediately after recording, slices were fixed for $2 \mathrm{~h}$ in $4 \%$ formaldehyde. Slices were preblocked for $2 \mathrm{~h}$ in PBS with $0.2 \%$ BSA and 5\% normal goat serum. Rabbit anti-tyrosine hydroxylase antibody (1:100) was then added and the slices agitated at $4^{\circ} \mathrm{C}$ for $48 \mathrm{~h}$. Slices were thoroughly rinsed with PBS and agitated with CY5 anti-rabbit secondary antibody (1:100) and fluorescein [dichlorotriazinylaminofluorescein (DTAF)]-conjugated streptavidin $(3.25 \mu \mathrm{l} / \mathrm{ml})$ at $4^{\circ} \mathrm{C}$ overnight. Cells were visualized with a Zeiss LSM 510 META microscope. Biocytin, BSA, and normal goat serum were obtained from Sigma Chemical. DTAF avidin was obtained from Jackson Immunoresearch, and rabbit anti-tyrosine hydroxylase antibody and CY5 anti-rabbit secondary antibody were obtained from Millipore Bioscience Research Reagents.

\section{Results}

\section{PHA-L injected into the VTA was detected in resident} neurons and fibers

We unilaterally injected the anterograde tracer PHA-L in the VTA to identify cellular compartments originating from resident neurons. For immunoelectron microscopy (see below, PHA-Llabeled axon terminals that either contain or lack VGluT2 labeling form synapses within the VTA), we analyzed three cases in which the PHA-L-immunoreactive cell bodies were confined to the PBP and PN of the VTA (Fig. 1). These cases were selected because we did not find PHA-L-immunoreactive cell bodies in areas rostral, dorsal, or lateral to the VTA, such as the lateral hypothalamic area and substantia nigra. In addition, in these cases we did not find immunoreactive cells bodies in the rostral linear nucleus or interfascicular nucleus, areas known to contain some TH neurons 
expressing VGluT2 transcripts (Kawano et al., 2006). Within the VTA, PHA-L-positive fibers were detected throughout the rostrocaudal and mediolateral aspects of the VTA. PHA-L-positive fibers were occasionally observed in the VTA contralateral to the injection site.

PHA-L-labeled axon terminals establish asymmetric or symmetric synapses with VTA neurons

Electron microscopic examination of VTA containing PHA-L immunoreactivity confined to the PBP and PN of the VTA showed PHA-L immunoreactivity in neuronal cell bodies, dendrites, axon terminals and glia (data not shown). PHA-L-positive axon terminals formed asymmetric (Fig. 2A) or symmetric (Fig. $2 B$ ) synapses, mostly with dendrites. We observed dense core vesicles in some of the PHA-L-positive axon terminals forming symmetric synapses (Fig. 2 B). Quantitative analysis of all PHA-L-positive terminals making recognizable synaptic contacts (Table 1) indicated that $50 \%$ of them established asymmetric synapses with dendrites and 9\% made asymmetric synapses with dendritic spines (Table 1). Less than half of all PHA-L-positive terminals (41\%) made symmetric synapses, all of which were onto dendrites, not on dendritic spines (Table 1).

PHA-L-labeled axon terminals that either contain or lack VGluT2 labeling form synapses within the VTA

We performed further neurochemical characterization of PHA-L-positive axon terminals to determine whether any of these terminals originated from VTA glutamatergic neurons. Ultrastructural analysis of VTA sections double labeled for the detection of PHA-L by immunoperoxidase activity and VGluT2 by immunogold-silver intensification revealed PHA-L-positive terminals containing VGluT2 [PHA-L $(+) /$ $\operatorname{VGluT2}(+)$ ] or lacking detectable levels of VGluT2 immunoreactivity [PHAL(+)/VGluT2(-)] (Fig. 3A). Quantitative analysis (Table 1 ) showed that $56 \%$ of all PHA-L-positive terminals forming synaptic contacts contained VGluT2. As expected of excitatory synapses, the vast majority of PHA-L $(+) /$ VGluT2 $(+)$ terminals formed asymmetric synapses (90\%). The PHA-L $(+) /$ VGluT2 $(+)$ terminals made synapses primarily on dendrites (Fig. 3 ), and occasionally on dendritic spines (Fig. 4A). A few PHA-L(+)/VGluT2(+) terminals appeared to form symmetric synapses on dendrites, but none were observed synapsing onto dendritic spines.

PHA-L-positive terminals that were VGluT2 negative [PHA$\mathrm{L}(+) /$ VGluT2 $(-)]$ constituted $44 \%$ of all PHA-L-positive ter-

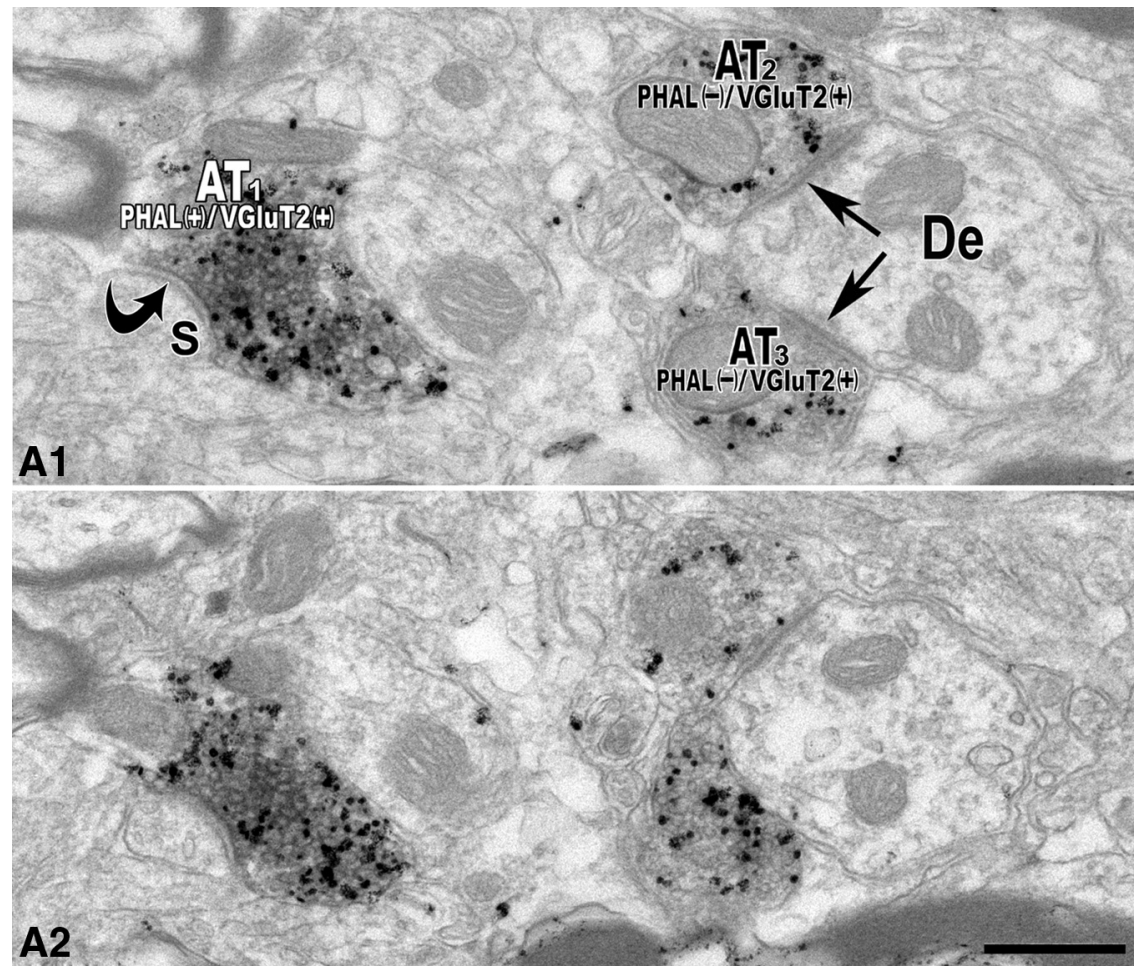

Figure 4. Electron micrographs of serial sections of a PHA-L/VGluT2 double-labeled axon terminal forming an asymmetric synapse with a dendritic spine of a local VTA neuron. A1, A2, Two consecutive sections showing a double-labeled $[\operatorname{PHAL}(+) /$ VGluT2 $(+)$ ] axon terminal $\left(\mathrm{AT}_{1}\right)$ that establishes an asymmetric synapse (curved black arrow) with a dendritic spine (S). Note that two axon terminals $\left(\mathrm{AT}_{2}\right.$ and $\mathrm{AT}_{3}$ ) lacking PHA-L but containing VGluT2 immunolabeling [PHAL $(-) / \mathrm{VGluT2}(+)$ ] form asymmetric synapses (black straight arrows) on the same dendrite (De). Scale bar (in A2): A1, A2, $0.5 \mu \mathrm{m}$.
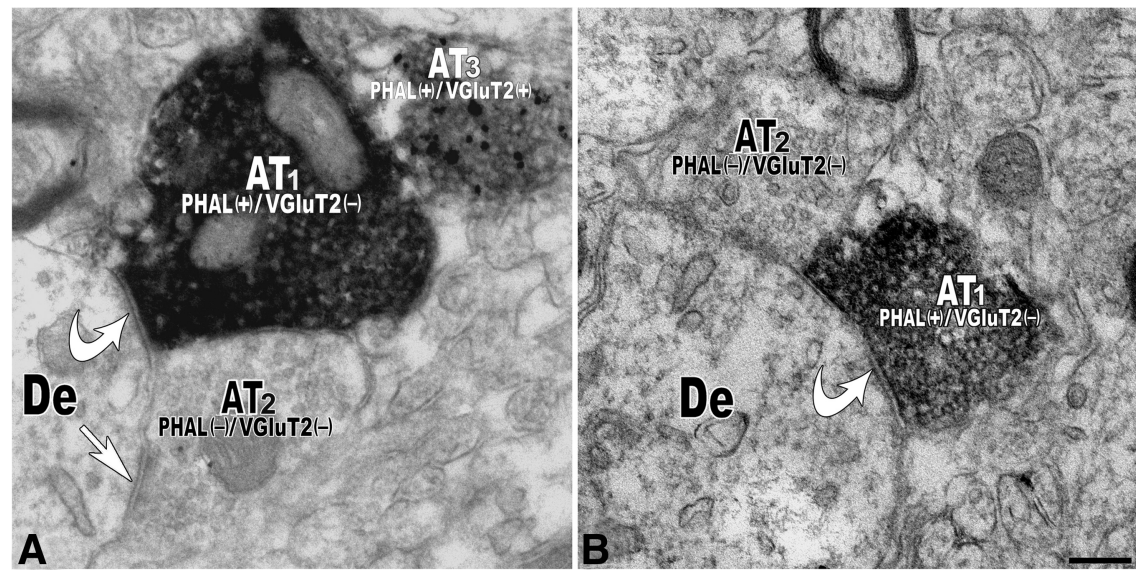

Figure 5. Electron micrographs showing PHA-L-labeled axon terminals lacking VGluT2 immunolabeling form symmetric synapses with dendrites of local VTA neurons. $\boldsymbol{A}$, Axon terminal $\left(\mathrm{AT}_{1}\right)$ containing PHA-L but lacking VGluT2 [PHAL $(+) /$ VGluT2 $\left.(-)\right]$ immunolabeling forms a symmetric synapse (white curved arrow) on a dendrite (De) receiving a symmetric synapse (white straight arrow) from an axon terminal $\left(\mathrm{AT}_{2}\right)$ lacking both PHA-L and VGluT2 immunoreactivity [PHAL( $(-) / \mathrm{VGluT2}(-)$ ]. Note a third axon terminal $\left(\mathrm{AT}_{3}\right)$ containing both PHA-L and VGluT2 labeling $[\mathrm{PHAL}(+) / \mathrm{VGluT2}(+)]$. B, Axon terminal $\left(\mathrm{AT}_{1}\right)$ containing PHA-L but lacking VGluT2 [PHAL(+)/VGluT2(-)] immunolabeling forms a symmetric synapse (white curved arrow) on a dendrite (De). Note an axon terminal $\left(\mathrm{AT}_{2}\right)$ lacking both PHA-L and VGluT2 immunoreactivity [PHAL(-)/VGluT2(-)]. Scale bar (in $\boldsymbol{B}$ ): $\boldsymbol{A}, \boldsymbol{B}, 0.2 \mu \mathrm{m}$. minals making discernable synaptic contacts. Seventy-nine percent of these PHA-L $(+) / V G l u T 2(-)$ axon terminals formed symmetric synapses with dendrites (Fig. $5 A, B$ ). The remaining PHA-L(+)/VGluT2(-) axon terminals established asymmetric synapses, mostly with dendrites, rarely with dendritic spines.

Because VTA neurons do not manufacture VGluT1, as a control we examined whether or not $\mathrm{PHA}-\mathrm{L}(+)$ axon terminals 


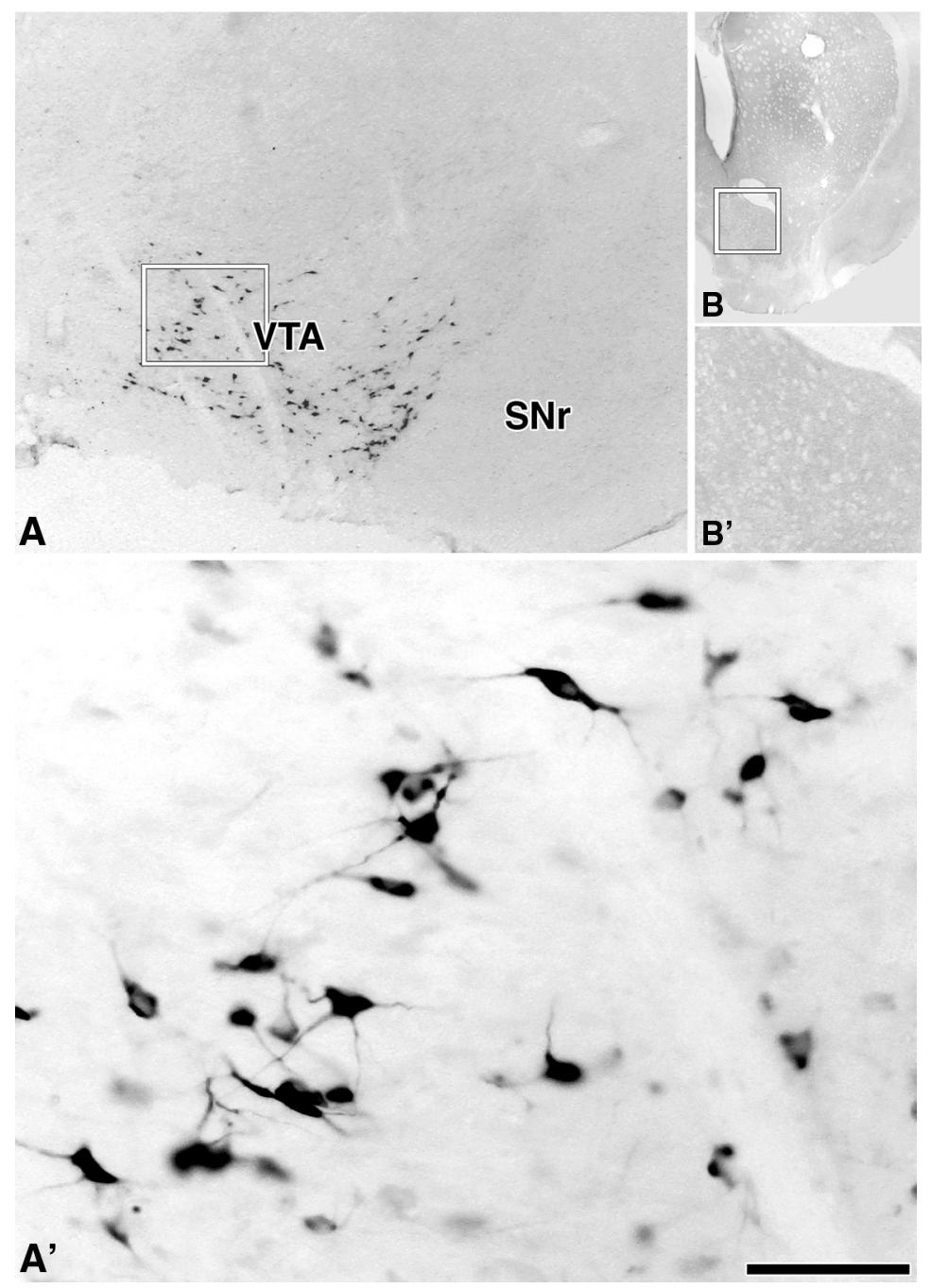

Figure 6. Detection of WGA in VTA neurons. $\boldsymbol{A}, \boldsymbol{A}^{\prime}$, WGA-immunoreactive neurons restricted to the VTA. Rectangle in $\boldsymbol{A}$ delimitates low-magnification area shown at higher magnification in $\boldsymbol{A}^{\prime} . \boldsymbol{B}, \boldsymbol{B}^{\prime}$, Ipsilateral striatum to the VTA shown in $\boldsymbol{A}$ and $\boldsymbol{A}^{\prime}$. Rectangle in $\boldsymbol{B}$ delimitates nucleus accumbens shown at higher magnification in $\boldsymbol{B}^{\prime}$; note lack of WGA-positive fibers. SNr, Substantia nigra reticulata. Scale bar (in $\boldsymbol{A}^{\prime}$ ): $580 \mu \mathrm{m}$ for $\boldsymbol{A} ; 75 \mu \mathrm{m}$ for $\boldsymbol{A}^{\prime} ; 2.5 \mathrm{~mm}$ for $\boldsymbol{B} ; 925 \mu \mathrm{m}$ for $\boldsymbol{B}^{\prime}$.

showing $>100$ and $<200$ transduced cells confined to the VTA (Figs. $6 A, A^{\prime}, 7 B, B^{\prime}$ ). We used anti-TH and anti-WGA antibodies in a double immunofluorescent labeling to determine whether the AAVWGAtr was able to transduce $\mathrm{TH}$-positive cells and TH-negative neurons (putative GABAergic or glutamatergic). Within the VTA, WGA was found in cells either containing or lacking $\mathrm{TH}$ immunoreactivity (Fig. 7C, $C^{\prime}$ ), indicating that under our experimental conditions the transduction is not neurochemically selective and raising the possibility we could use the WGA to detect VTA glutamatergic terminals.

WGA labeling was not detected in cell bodies outside the VTA, suggesting that transduction was limited to cell bodies local to the injection site and that the virus was not taken up by terminals and transported to cell bodies outside of the VTA (Fig. 6B, $B^{\prime}$; supplemental Fig. 1, available at www.jneurosci.org as supplemental material). Surprisingly, we did not detect WGA in VTA projection targets. This lack of detection of WGA immunoreactivity in VTA target areas, such as the nucleus accumbens, was unexpected. We therefore also injected AAV-WGAtr into other brain structures and found that the WGA detection was similarly restricted to local cell bodies and processes. We therefore speculate that the restricted detection of truncated WGA may result from molecular trafficking differences between fibers that terminate proximal to the soma and fibers that project to other brain regions.

To further validate the specificity of the AAV-WGAtr for labeling terminals arising from local neurons, we performed ultrastructural analysis of double WGA/ VGluT1 immunolabeled tissue. VGluT1

forming asymmetric synapses contain VGluT1. We performed ultrastructural analysis of double PHA-L/VGluT1 immunolabeled VTA, using brain sections from the same three rats in which we detected PHA-L/VGluT2 immunolabeling. In contrast to the above-described coexistence of PHA-L/VGluT2 immunolabeling in axon terminals, VGluT1 labeling was often found in axon terminals making asymmetric synapses but lacking PHA-L immunoreactivity. Of the 60 examined PHA-L-positive axon terminals making asymmetric synapses on dendrites, none of them contained VGluT1 immunoreactivity.

\section{AAV-WGAtr injected into the VTA transduced resident neurons containing or lacking TH immunoreactivity} To address the question of local glutamatergic connections in the VTA using a different anatomical tool, we developed an AAV that causes transduced neurons to express a truncated form of WGA. We then injected the AAV-WGAtr into the VTA of a different group of rats. These injections resulted in expression of WGA in cell bodies and fibers within the VTA (Fig. 6A, $A^{\prime}$ ). By intra-VTA delivery of different amounts of viral particles, we varied the number of transduced cells. We analyzed sections from brains labeling was seen only in axon terminals lacking WGA immunoreactivity. Further, among the 22 examined WGA-immunopositive axon terminals that made asymmetric synapses, none of them contained VGluT1 immunoreactivity. This is consistent with our PHA-L/VGluT1 analysis, and suggests that WGA labeling indicates a terminal arising from a transduced VTA neuron.

\section{WGA-labeled axon terminals establish asymmetric or symmetric synapses with VTA neurons}

We analyzed three cases in which the WGA-immunoreactive cell bodies were confined to the PBP and PN of the VTA. Similar to the PHA-L-labeled axon terminals, the WGA-positive axon terminals formed symmetric (Fig. 8A) or asymmetric (Fig. 8C) synapses. Ultrastructural analysis of VTA sections double labeled for the detection of WGA by immunoperoxidase activity and VGluT2 by immunogold-silver intensification revealed WGApositive terminals that either lacked VGluT2 [WGA $(+) /$ VGluT2(-)] (Fig. 8A) or contained VGluT2 immunoreactivity [WGA $(+) / \operatorname{VGluT2}(+)$ ] (Fig. 8B,C). Quantitative analysis (Tables 2,3 ) showed that $55 \%$ of all WGA-positive terminals forming synaptic contacts contained VGluT2. In agreement with the 
putative excitatory nature of asymmetric synapses, the vast majority of $\mathrm{WGA}(+) /$ VGluT2 $(+)$ terminals formed asymmetric synapses $(92 \%)$.

WGA-positive terminals that were VGluT2 negative [WGA(+)/VGluT2(-)] constituted $45 \%$ of all WGA-positive terminals making recognizable synaptic contacts (Table 2). These included both symmetric and asymmetric synapses.

\section{PHA-L-labeled axon terminals} containing VGluT2 form synaptic contacts on TH-immunopositive and TH-immunonegative dendrites To determine the neurochemical identity of the postsynaptic targets of local VTA glutamatergic terminals, we used tissue from PHA-L-injected VTA to perform triple immunocytochemical ultrastructural studies to determine the neurochemical identity of the postsynaptic targets of local VTA glutamatergic terminals. For the triple labeling procedure we took advantage of the minimally overlapping ultrastructural distribution of VGluT2 and $\mathrm{TH}$ within the VTA: while VGluT2 protein is confined to presynaptic compartments, $\mathrm{TH}$ is almost exclusively localized postsynaptically in dendrites and somata (Bayer and Pickel, 1991; Carr and Sesack, 2000; Omelchenko and Sesack, 2005). This segregation allows the detection of presynaptic VGluT2 simultaneously with postsynaptic TH by immunogold-silver intensification, and PHA-L by immunoperoxidase activity.

We observed some double-labeled PHA-L $(+) / \operatorname{VGluT} 2(+)$ axon terminals that made asymmetric synapses on dendrites containing detectable levels of TH immunolabeling (Fig. 9A1,A2) and some that made asymmetric synapses on dendrites lacking detectable $\mathrm{TH}$ immunoreactivity (Fig. 9B1,B2). In addition, we detected some PHA-L(+)/VGluT2(-) terminals that made synaptic contacts on both TH-immunopositive and TH-immunonegative dendrites (data not shown). Therefore, it is likely that at least some VTA DAergic neurons receive synaptic inputs from VTA glutamatergic neurons.

\section{Blocking action potential activity decreases the frequency of} excitatory events in VTA neurons, in vitro electrophysiology Given these anatomical results, and because many VTA neurons, including non-DAergic neurons, fire spontaneously in the slice, we hypothesized that some glutamate-mediated spontaneous EPSCs detected in VTA neurons would be driven by action potential activity of local glutamatergic neurons. We tested for the presence of local, functional glutamatergic synapses using whole-cell recordings to detect AMPAR-mediated currents in acute horizontal rat brain slices. Care was taken to remove possible sources of glutamate outside of the VTA when blocking the slice preparation (supplemental Fig. 2, available at www.jneurosci.org as supplemental material). Spontaneously occurring events can be voltage and $\mathrm{Ca}^{2+}$ dependent (Del Castillo and Katz, 1954; Boyd and Martin,
1956; Liley, 1956; Hubbard, 1961); however, there are also often voltage-independent events (Del Castillo and Katz, 1954; Hubbard, 1961; Katz and Miledi, 1963; Elmqvist and Feldman, 1965). Therefore, spontaneous events can include both action potentialdriven and action potential-independent events. A decrease in event frequency in a slice preparation following blockade of action potential activity suggests that a portion of the spontaneous events were driven by locally firing neurons. Since action potential-independent spontaneous glutamatergic events are present in most VTA neurons (Margolis et al., 2005; Deng et al., 2009; Xiao et al., 2009), we should observe a decrease in frequency, but not elimination, of glutamatergic events when action potential activity is blocked by TTX.

All recordings were made in VTA neurons that expressed the hyperpolarization-activated cation current, $I_{\mathrm{h}}$, many of which were DAergic neurons, as confirmed by $\mathrm{TH}$ immunocytochemistry following recording. We recorded spontaneously occurring EPSCs. Excitatory synaptic input was isolated from inhibitory input by pharmacologically blocking $\mathrm{GABA}_{\mathrm{A}}$ receptors with bath application of the antagonist picrotoxin $(100 \mu \mathrm{M})$. At the end of a subset of experiments the non-NMDA glutamate receptor antagonist DNQX $(10 \mu \mathrm{M})$ blocked all remaining synaptic signaling (Fig. 10F), suggesting that the events recorded in the presence of picrotoxin resulted from glutamatergic activation of AMPARs. 

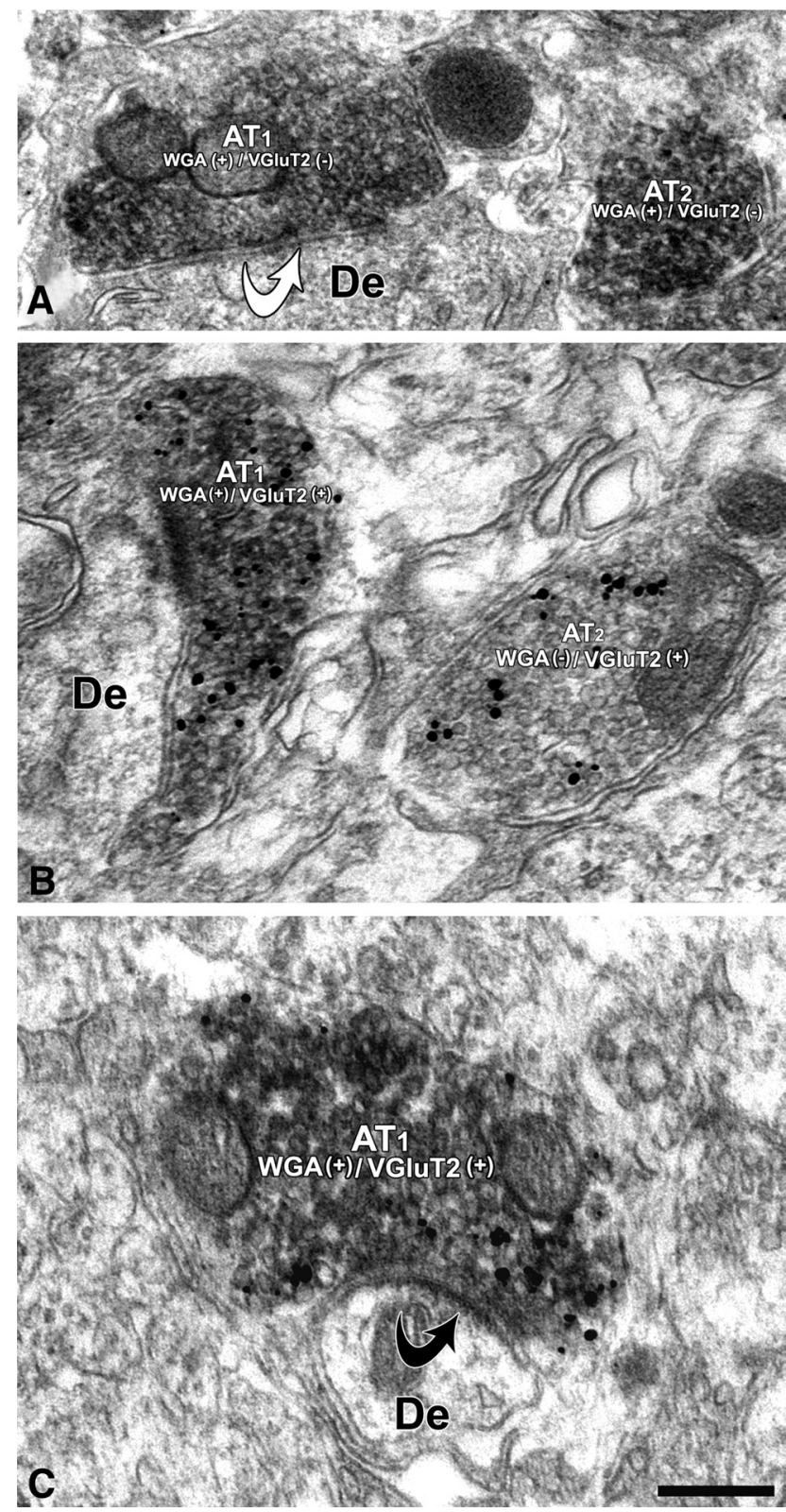

Figure 8. Electron micrographs of WGA-labeled axon terminals forming asymmetric or symmetric synapses on dendrites in the VTA. $\boldsymbol{A}$, Two axon terminals ( $\mathrm{AT}_{1}$ and $\mathrm{AT}_{2}$ ) containing WGA immunolabeling $[W G A(+)]$, one of which $\left(A T_{1}\right)$ forms a symmetric synapse (white curved arrow) with a dendrite (De). $\boldsymbol{B}$, Two axon terminals ( $\mathrm{AT}_{1}$ and $\mathrm{AT}_{2}$ ) containing VGluT2 immunolabeling [immunogold-silver particles, VGluT2 $(+)$ ]. While the $\mathrm{AT}_{1}$ also contains WGA immunolabeling [immunoperoxidase product, WGA(+)], the $\mathrm{AT}_{2}$ lacks WGA immunolabeling [WG $(-)]$. C, An axon terminal $\left(\mathrm{AT}_{1}\right)$ containing both WGA and VGluT2 immunolabeling $[W G A(+) /$ VGluT2 $(+)]$ forms an asymmetric synapse (white curved arrow) with a dendrite (De). Scale bar (in C): $0.4 \mu \mathrm{m}$ for $\boldsymbol{A} ; 0.3 \mu \mathrm{m}$ for $\boldsymbol{B}$; and $0.2 \mu \mathrm{m}$ for $\boldsymbol{C}$.

Table 2. Neurochemical composition of WGA-labeled axon terminals in the rat VTA: VGluT2 signal

\begin{tabular}{lll}
\hline & $\begin{array}{l}\text { WGA-labeled terminals } \\
\text { with VGluT2 signal }\end{array}$ & $\begin{array}{l}\text { WGA-labeled terminals } \\
\text { without VGluT2 signal }\end{array}$ \\
\hline $\begin{array}{l}\text { Total number of WGA-labeled terminals } \\
\quad 4100 \%(n=124)]\end{array}$ & $52 \%(n=60)$ & $54)$ \\
$\begin{array}{c}\text { Total number of WGA-labeled terminals } \\
\text { making recognizable synapses }\end{array}$ & $55 \%(n=26)$ & $45 \%(n=21)$ \\
$\quad[100 \%(n=47)]$ & & \\
\hline
\end{tabular}

Table 3. Neurochemical composition of WGA-labeled axon terminals in the rat VTA: synapse formation

\begin{tabular}{lll}
\hline & $\begin{array}{l}\text { WGA-labeled terminals } \\
\text { making recognizable } \\
\text { asymmetric synapses }\end{array}$ & $\begin{array}{l}\text { WGA-labeled terminals } \\
\text { making recognizable } \\
\text { symmetric synapses }\end{array}$ \\
\hline $\begin{array}{c}\text { WGA-labeled terminals with VGluT2 } \\
\text { signal }[100 \%(n=26)]\end{array}$ & $92 \%(n=24)$ & $8 \%(n=2)$ \\
$\begin{array}{l}\text { WGA-labeled terminals without } \\
\text { VGluT2 signal [100\% }(n=21)]\end{array}$ & $24 \%(n=5)$ & $76 \%(n=16)$ \\
\hline
\end{tabular}

We then compared the baseline frequency of spontaneous EPSCs to that observed during bath application of the $\mathrm{Na}^{+}$channel blocker TTX (500 nM) to terminate action potential firing. The baseline frequency of EPSCs in control ACSF was $2.0 \pm 0.4 \mathrm{~Hz}$. When TTX was added to the perfusion solution, the frequency of EPSCs decreased by an average of 29\% (range: $2-36 \% ; n=6 ; p<$ 0.05 Student's $t$ test) (Fig. $10 F$ ). Because the baseline frequency of events was low in control ACSF, we conducted additional experiments in which we depolarized the slices by increasing the external concentration of $\mathrm{K}^{+}$to $5 \mathrm{~mm}$. Depolarizing the slices in this way will increase both the action potential-dependent and independent EPSCs by increasing cell firing and depolarizing axon terminals. In these experiments, the baseline EPSC frequency was $\sim 3$ times greater than in control ACSF, and TTX application similarly decreased the frequency of EPSCs by a mean of $26 \%$ (range: $6-64 \% ; n=10 ; p<0.005$, Student's $t$ test) (Fig. $10 F)$. In immunocytochemically identified neurons, the frequency of spontaneous EPSCs decreased in both $\mathrm{TH}$-immunopositive (in 5 mM K ${ }^{+}$; range: $12-30 \% ; n=3$ ) and $\mathrm{TH}$-immunonegative (in 5 $\mathrm{mM} \mathrm{K}^{+}$; range: $6-34 \% ; n=3$ ) neurons (Fig. $10 H$ ). In experiments conducted in elevated $\mathrm{K}^{+}$, there was a very small (5\%) but significant decrease in the amplitude of EPSCs with TTX application ( $n=10 ; p<0.05$, Student's $t$ test) (Fig. $10 E)$. A trend toward such an amplitude change with TTX application was observed in both $\mathrm{TH}$-immunopositive and $\mathrm{TH}$-immunonegative neurons (Fig. 10G). These data support the conclusion that the anatomically identified synapses onto VTA neurons from VTA glutamatergic neurons are functional.

\section{Discussion}

We found that VTA glutamatergic neurons make local synapses on both DAergic and non-DAergic VTA neurons. We provide both anatomical and functional evidence for these connections by using ultrastructural and electrophysiological techniques. Our ultrastructural findings also indicate that VTA nonglutamatergic neurons make symmetric synaptic contacts with other VTA neurons; these terminals likely arise from VTA GABAergic neurons.

\section{Identification of synaptic terminals from resident}

glutamatergic and nonglutamatergic neurons in the VTA

We established the VTA origin of identified glutamatergic synapses using 2 anatomical procedures: a traditional approach (local cellular uptake of PHA-L) and a novel procedure (local cellular synthesis and corresponding intracellular accumulation of WGA). PHA-L is a sensitive anterograde tracer that is incorporated by neurons at the injection site with an insignificant uptake by fibers of passage and negligible retrograde axonal transport (Gerfen and Sawchenko, 1984; Deller et al., 2000). Since local glutamatergic connections are rarely reported, it was important to replicate observation obtained with PHA-L with an alternative method. Thus we used intra-VTA injection of AAV- 
WGAtr that resulted in expression of WGA in cell bodies and fibers within the VTA.

To support the premises that PHA-L was selectively incorporated by local VTA neurons and that WGA synthesis and accumulation were limited to resident VTA neurons, we examined the degree of expression of VGluT1 immunoreactivity within PHA-L- or WGA-containing axon terminals. We chose VGluT1 as control because VTA neurons do not express VGluT1 mRNA (Kawano et al., 2006; Yamaguchi et al., 2007), and thus axon terminals containing VGluT1, which are not uncommon in the VTA (Omelchenko and Sesack, 2007), have an exclusively extrinsic origin. In agreement with this notion, we did not detect VGluT1 immunoreactivity in terminals containing either PHA-L or WGA.

In the analysis of ultrastructural data, asymmetric and symmetric synapses are generally considered to be excitatory and inhibitory, respectively. However, we found that not all PHA-L-positive terminals making asymmetric synapses contained VGluT2 immunolabeling. This lack of VGluT2 signal in these terminals

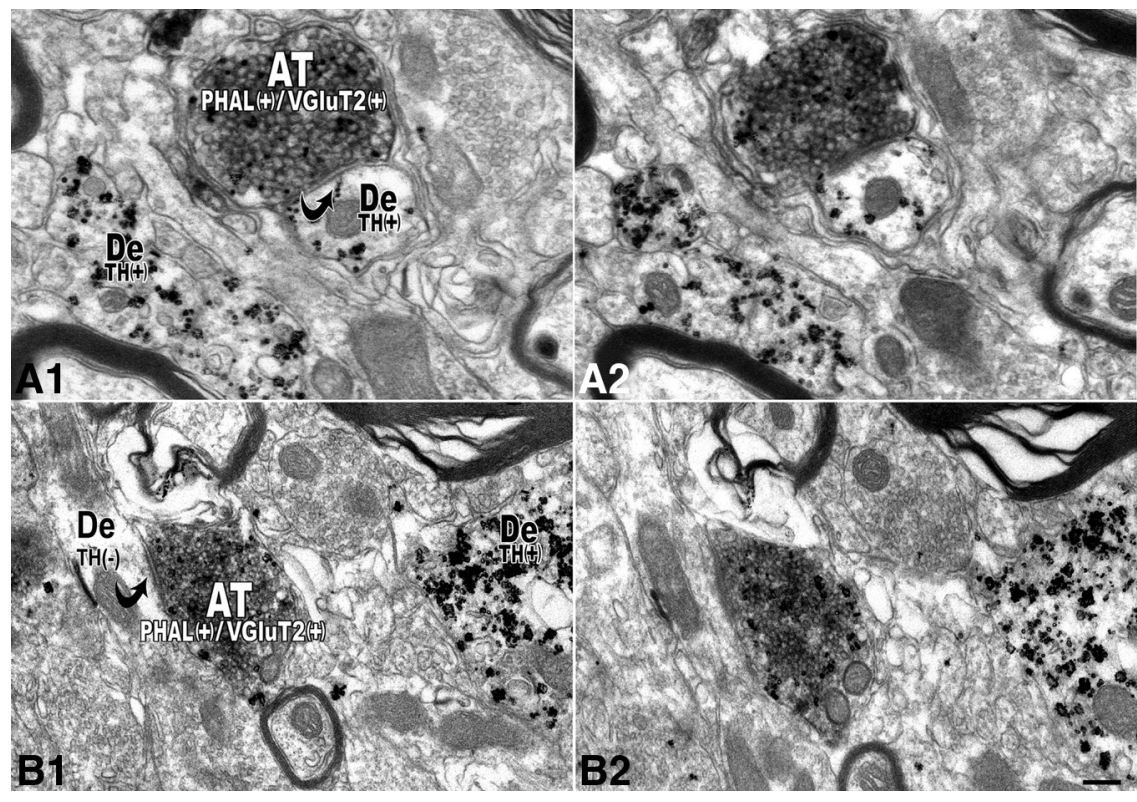

Figure 9. Electron micrographs showing double-labeled PHA-L/VGluT2 axon terminals establishing asymmetric synapses with VTA neurons containing or lacking TH. A1, A2, Two consecutive sections showing that an axon terminal containing PHA-L (peroxidase product) and VGluT2 (immunogold-silver particles) $[\mathrm{PHAL}(+) / \mathrm{VGluT2}(+)]$ establishes an asymmetric synapse (black curved arrow in $\mathbf{A 1}$ ) with a dendrite (De) containing TH immunolabeling (immunogold-silver particles) $[\mathrm{TH}(+)]$. B1, B2, Two consecutive sections of an axon terminal containing PHA-L (peroxidase product) and VGluT2 (immunogold-silver particles) [PHAL $(+) /$ VGluT2 $(+)]$ establishes an asymmetric synapse (black curved arrow in B1) with a dendrite lacking TH immunolabeling [TH( - )]. Scale bar (in B2): $0.2 \mu \mathrm{m}$ for $\boldsymbol{A 1}, \boldsymbol{A 2} ; 0.3 \mu \mathrm{m}$ for $\boldsymbol{B 1}, \boldsymbol{B}$.

may be due to a failure to detect VGluT2 in some terminals, or the possibility that these terminals may derive from intrinsic GABAergic or DAergic neurons with the capability of making asymmetric synapses. GABAergic axon terminals that form asymmetric synapses have been found in the substantia nigra (Ribak et al., 1976; Nitsch and Riesenberg, 1988; Bolam and Smith, 1990), and a small number of TH-positive terminals making asymmetric synapses has been observed in the VTA (Bayer and Pickel, 1990). Although symmetric synaptic structure is usually associated with inhibitory synapses, we occasionally found PHA-L $(+)$ /VGluT2 $(+)$ terminals forming symmetric synapses on dendrites. This is consistent with a previous report of VGluT2expressing axon terminals forming symmetric contacts in the VTA (Omelchenko and Sesack, 2007).

We provide electrophysiological evidence that the local glutamatergic terminals release neurotransmitter in an action potential-dependent manner by showing that the frequency of spontaneous EPSCs in both DAergic and non-DAergic VTA neurons decreases when action potential activity is blocked. We observed, on average, a $30 \%$ decrease in frequency of spontaneous EPSCs in VTA neurons in response to TTX application. The remaining events are likely due to vesicle fusion events that are independent of action potential-induced depolarization of the axon terminal. Such action potential-independent events have been observed in many parts of the CNS (Hubbard, 1961; Farrant and Cull-Candy, 1991; Staley and Mody, 1991), including at both glutamate and GABA synapses in the VTA (Margolis et al., 2005, 2008; Xiao et al., 2008, 2009; Deng et al., 2009). We observed a slight decrease in the amplitude of spontaneous EPSCs following the application of TTX. Since electrical excitation of the presynaptic terminal, such as by action potential propagation, could evoke the simultaneous release of more than one vesicle, it is possible that the decrease in amplitude is due to the TTX application diminishing the frequency of simultaneous vesicle fusions.
It is interesting to note that similar to the ultrastructural analysis, both $\mathrm{TH}$ immunopositive and immunonegative VTA neurons displayed electrophysiological evidence that they receive inputs from local glutamatergic neurons.

VTA non-DAergic and DAergic neurons are under dual local circuit control: glutamatergic and GABAergic

It is generally accepted that intrinsic GABAergic neurons synapse onto projecting DAergic neurons. In agreement with this idea, we found that approximately half of the total population of either PHA-L or WGA axon terminals from tagged VTA neurons makes symmetric (putative inhibitory) synapses on local dendrites. We infer that the PHA-L or WGA axon terminals making local symmetric synapses are GABAergic because they lack VGluT2 or VGluT1, and multiple ultrastructural studies have shown that axon terminals of symmetric synapses are GABAergic in many brain areas, including the VTA (Bayer and Pickel, 1991; Omelchenko and Sesack, 2005). Moreover, a recent ultrastructural study using PHA-L tagging of VTA fibers and immunolabeling for GABA demonstrated that VTA GABAergic neurons form synapses on local DAergic neurons (Omelchenko and Sesack, 2009). Functional evidence for local connections arising from VTA GABAergic neurons comes from in vitro electrophysiological studies showing that there are TTX-sensitive inhibitory PSCs in putative DAergic neurons (Johnson and North, 1992a). It is not clear whether or not these GABAergic inputs arise from interneurons lacking axonal projections outside of the VTA.

Given the current model of intra-VTA circuitry and the prominent role ascribed to local GABAergic connections in the VTA, it is noteworthy that in the present study we found that only half of the total population of PHA-L or WGA axon terminals forming synaptic contacts make symmetric synapses on VTA dendrites. Moreover, the vast majority of PHA-L or WGA axon terminals making local asymmetric synapses are glutamatergic, as they con- 
A

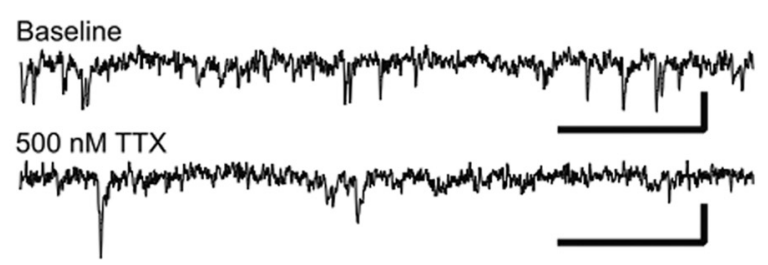

B

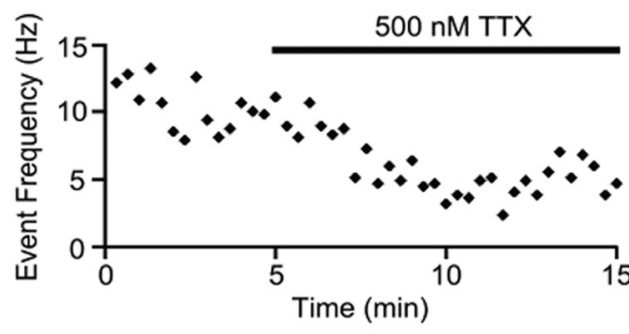

$\mathrm{C}$
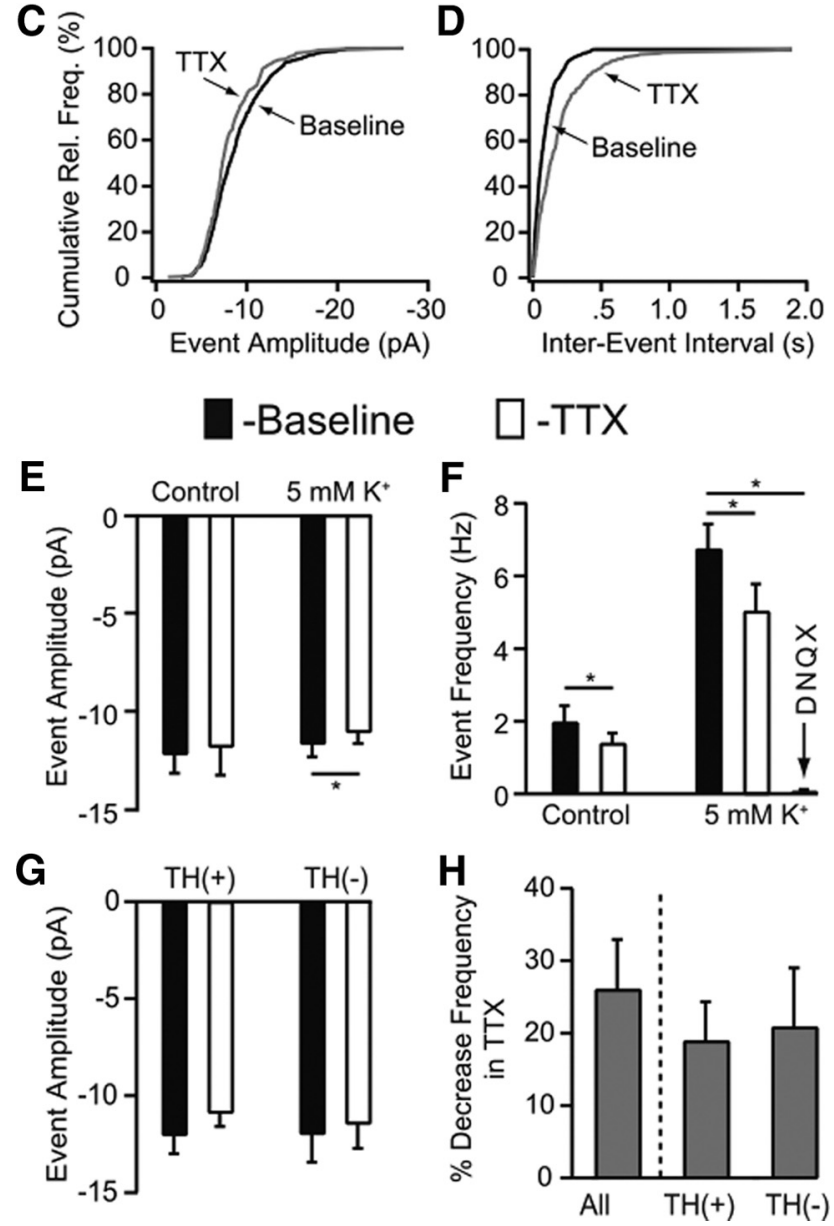

Figure 10. Blocking action potential activity decreases the frequency of excitatory events in VTA whole-cell slice recordings. $\boldsymbol{A}, \boldsymbol{B}$, Sample traces (calibration in $\boldsymbol{A}, 10 \mathrm{pA}$ and $100 \mathrm{~ms}$ ) and time course $(\boldsymbol{B})$ of spontaneous glutamatergic events recorded in one neuron under baseline conditions and in the presence of $500 \mathrm{~nm}$ TTX in a slice depolarized with $5 \mathrm{~mm} \mathrm{~K}^{+}$. $\boldsymbol{B}$, Inset, This recording was made in an $I_{\mathrm{h}}(+)$ neuron (calibration, $200 \mathrm{pA}$ and $\left.50 \mathrm{~ms}\right)$. C, D, For this neuron, the cumulative event plots show that in TTX the amplitudes of spontaneous events slightly decreased ( $\boldsymbol{C}$, while the number of longer interevent intervals greatly increased (D). $\boldsymbol{E}$, Across cells, $500 \mathrm{~nm}$ TTX decreased the amplitude of excitatory events only in $5 \mathrm{~mm} \mathrm{~K}^{+}(n=10, p<$ 0.05 ) but not in control ACSF $(n=6, p>0.05)$. $\boldsymbol{F}$, There was a significant decrease in the frequency of spontaneous excitatory events with $500 \mathrm{~nm}$ TTX application both in control ACSF $(n=6)$ and in $5 \mathrm{~mm} \mathrm{~K}^{+}(n=10)$. Application of the AMPAR antagonist DNQX (10 $\left.\mu \mathrm{M}\right)$ decreased the excitatory event frequency to approximately zero $(n=4) . \mathbf{G}$, In neurons directly identified as TH positive $(n=3)$ or TH negative $(n=3)$, in $5 \mathrm{~mm} \mathrm{~K}^{+}$there was a trend toward a decrease in excitatory event amplitude with bath application of $500 \mathrm{~nm} \mathrm{TTX.} \boldsymbol{H}, \ln 5 \mathrm{~mm} \mathrm{~K}{ }^{+}$, tain VGluT2 immunoreactivity. These anatomical observations raise the possibility that the extent of modulation of VTA neurons by local glutamatergic connections rivals that of intrinsic GABAergic neurons. While we show that VTA neurons containing VGluT2 establish local synapses, it is not clear whether or not these neurons are interneurons lacking axonal projections outside of the VTA. To date, the septum is the only brain region in which synapses arising from local VGluT2-expressing neurons has been documented (Hajszan et al., 2004; Manseau et al., 2005).

Finally, since we found some VGluT2-positive contacts on $\mathrm{TH}$-positive cells and others on TH-negative cells, and similarly, some VGluT2-negative contacts on TH-positive cells and others on TH-negative cells, we propose that resident glutamatergic and GABAergic neurons provide synaptic inputs to DAergic, GABAergic, and glutamatergic neurons within the VTA.

Possible functional implications of interactions between VTA glutamatergic and DAergic neurons

The VTA is thought to play a role in goal-directed behavior and in the reward processing of natural rewards and of several drugs of abuse (Schultz, 2002; Wise, 2004). The VTA dopamine neurons have two firing patterns that contribute to mesolimbic DA neurotransmission; a tonic DA release mediated by pacemaker-like DA neural activity, and a phasic DA release mediated by burst firing (Grace, 1991; Floresco et al., 2003; Sombers et al., 2009). The nonlinear increase in synaptic DA release induced by burst firing of DA neurons (Chergui et al., 1994) is considered to be functionally relevant for signaling salient environmental stimuli (Grace, 1991; Overton and Clark, 1992; Berridge and Robinson, 1998; Schultz, 1998; Cooper, 2002). Excitatory glutamatergic inputs to midbrain DAergic neurons are necessary for inducing burst firing (Grace and Bunney, 1984; Overton and Clark, 1992; Chergui et al., 1994; Paladini et al., 1999; Floresco et al., 2003) and DA release in the nucleus accumbens (Sombers et al., 2009). It has recently been suggested that the laterodorsal tegmental nucleus (LDTg) is essential to allow glutamate to elicit burst firing of VTA neurons, as the inactivation of the LDTg prevents glutamate from inducing burst firing of VTA neurons (Lodge and Grace, 2006). While it remains to be determined whether intrinsic glutamatergic neurons play a role in VTA neurons burst firing, the local glutamatergic synapses reported here raise this intriguing possibility.

It has recently been suggested that VTA glutamatergic and DAergic neurons projecting to the PFC may provide both transient reward-related information via glutamatergic signaling as well as exert state-dependent control of PFC networks via DA release (Lavin et al., 2005). Anatomical studies provide evidence for reciprocal connectivity between VTA DAergic neurons and principal neurons in the PFC (Carr and Sesack, 2000). In addition, recent observations have shown that VTA neurons expressing VGluT2 mRNA project to the PFC (Hur and Zaborszky, 2005). Therefore, VTA glutamatergic neurons may regulate activity of DA neurons directly through local synaptic connections or indirectly by signaling on PFC neurons that provide feedback to VTA DA neurons.

In conclusion, we demonstrate here that VTA glutamatergic and nonglutamatergic neurons establish local synaptic connections. The targets of these local synapses include both DAergic

$\leftarrow$

there was a decrease in excitatory event frequency across all neurons tested, and this decrease occurred in both TH-positive and TH-negative neurons. ${ }^{*} p<0.05$. 
and non-DAergic VTA neurons. This novel type of local communication expands the modalities of local signaling in the VTA to now include glutamatergic, GABA, and DA, each of which likely modulates both DAergic and non-DAergic projection neurons.

\section{References}

Bai L, Xu H, Collins JF, Ghishan FK (2001) Molecular and functional analysis of a novel neuronal vesicular glutamate transporter. J Biol Chem 276:36764-36769.

Bayer VE, Pickel VM (1990) Ultrastructural localization of tyrosine hydroxylase in the rat ventral tegmental area: relationship between immunolabeling density and neuronal associations. J Neurosci 10:2996-3013.

Bayer VE, Pickel VM (1991) GABA-labeled terminals form proportionally more synapses with dopaminergic neurons containing low densities of tyrosine hydroxylase-immunoreactivity in rat ventral tegmental area. Brain Res 559:44-55.

Bellocchio EE, Hu H, Pohorille A, Chan J, Pickel VM, Edwards RH (1998) The localization of the brain-specific inorganic phosphate transporter suggests a specific presynaptic role in glutamatergic transmission. J Neurosci 18:8648-8659.

Berridge KC, Robinson TE (1998) What is the role of dopamine in reward: hedonic impact, reward learning, or incentive salience? Brain Res Brain Res Rev 28:309-369.

Bolam JP, Smith Y (1990) The GABA and substance P input to dopaminergic neurones in the substantia nigra of the rat. Brain Res 529:57-78.

Boyd IA, Martin AR (1956) The end-plate potential in mammalian muscle. J Physiol 132:74-91.

Cameron DL, Williams JT (1993) Dopamine D1 receptors facilitate transmitter release. Nature 366:344-347.

Carr DB, Sesack SR (2000) Projections from the rat prefrontal cortex to the ventral tegmental area: target specificity in the synaptic associations with mesoaccumbens and mesocortical neurons. J Neurosci 20:3864-3873.

Chergui K, Suaud-Chagny MF, Gonon F (1994) Nonlinear relationship between impulse flow, dopamine release and dopamine elimination in the rat brain in vivo. Neuroscience 62:641-645.

Chuhma N, Zhang H, Masson J, Zhuang X, Sulzer D, Hen R, Rayport S (2004) Dopamine neurons mediate a fast excitatory signal via their glutamatergic synapses. J Neurosci 24:972-981.

Cooper DC (2002) The significance of action potential bursting in the brain reward circuit. Neurochem Int 41:333-340.

Del Castillo J, Katz B (1954) Changes in end-plate activity produced by presynaptic polarization. J Physiol 124:586-604.

Deller T, Naumann T, Frotscher M (2000) Retrograde and anterograde tracing combined with transmitter identification and electron microscopy. J Neurosci Methods 103:117-126.

Deng C, Li KY, Zhou C, YeJH (2009) Ethanol enhances glutamate transmission by retrograde dopamine signaling in a postsynaptic neuron/synaptic bouton preparation from the ventral tegmental area. Neuropsychopharmacology 34:1233-1244.

Elmqvist D, Feldman DS (1965) Spontaneous activity at a mammalian neuromuscular junction in tetrodotoxin. Acta Physiol Scand 64:475-476.

Fallon JH, Schmued LC, Wang C, Miller R, Banales G (1984) Neurons in the ventral tegmentum have separate populations projecting to telencephalon and inferior olive, are histochemically different, and may receive direct visual input. Brain Res 321:332-336.

Farrant M, Cull-Candy SG (1991) Excitatory amino acid receptor-channels in Purkinje cells in thin cerebellar slices. Proc Biol Sci 244:179-184.

Floresco SB, West AR, Ash B, Moore H, Grace AA (2003) Afferent modulation of dopamine neuron firing differentially regulates tonic and phasic dopamine transmission. Nat Neurosci 6:968-973.

Fremeau RT Jr, Troyer MD, Pahner I, Nygaard GO, Tran CH, Reimer RJ, Bellocchio EE, Fortin D, Storm-Mathisen J, Edwards RH (2001) The expression of vesicular glutamate transporters defines two classes of excitatory synapse. Neuron 31:247-260.

Fujiyama F, Furuta T, Kaneko T (2001) Immunocytochemical localization of candidates for vesicular glutamate transporters in the rat cerebral cortex. J Comp Neurol 435:379-387.

Gao G, Vandenberghe LH, Alvira MR, Lu Y, Calcedo R, Zhou X, Wilson JM (2004) Clades of Adeno-associated viruses are widely disseminated in human tissues. J Virol 78:6381-6388.

Gerfen CR, Sawchenko PE (1984) An anterograde neuroanatomical tracing method that shows the detailed morphology of neurons, their axons and terminals: immunohistochemical localization of an axonally transported plant lectin, Phaseolus vulgaris leucoagglutinin (PHA-L). Brain Res 290:219-238

Grace AA (1991) Phasic versus tonic dopamine release and the modulation of dopamine system responsivity: a hypothesis for the etiology of schizophrenia. Neuroscience 41:1-24.

Grace AA, Bunney BS (1984) The control of firing pattern in nigral dopamine neurons: burst firing. J Neurosci 4:2877-2890.

Hajszan T, Alreja M, Leranth C (2004) Intrinsic vesicular glutamate transporter 2-immunoreactive input to septohippocampal parvalbumincontaining neurons: novel glutamatergic local circuit cells. Hippocampus 14:499-509.

Hayashi M, Otsuka M, Morimoto R, Hirota S, Yatsushiro S, Takeda J, Yamamoto A, Moriyama Y (2001) Differentiation-associated Na+dependent inorganic phosphate cotransporter (DNPI) is a vesicular glutamate transporter in endocrine glutamatergic systems. J Biol Chem 276:43400-43406.

Herzog E, Bellenchi GC, Gras C, Bernard V, Ravassard P, Bedet C, Gasnier B, Giros B, El Mestikawy S (2001) The existence of a second vesicular glutamate transporter specifies subpopulations of glutamatergic neurons. J Neurosci 21:RC181.

Howard DB, Powers K, Wang Y, Harvey BK (2008) Tropism and toxicity of adeno-associated viral vector serotypes 1, 2, 5, 6, 7, 8, and 9 in rat neurons and glia in vitro. Virology 372:24-34.

Hubbard JI (1961) The effect of calcium and magnesium on the spontaneous release of transmitter from mammalian motor nerve endings. J Physiol 159:507-517.

Hur EE, Zaborszky L (2005) Vglut2 afferents to the medial prefrontal and primary somatosensory cortices: a combined retrograde tracing in situ hybridization study [corrected]. J Comp Neurol 483:351-373.

Johnson SW, North RA (1992a) Opioids excite dopamine neurons by hyperpolarization of local interneurons. J Neurosci 12:483-488.

Johnson SW, North RA (1992b) Two types of neurone in the rat ventral tegmental area and their synaptic inputs. J Physiol 450:455-468.

Katz B, Miledi R (1963) A study of spontaneous miniature potentials in spinal motoneurones. J Physiol 168:389-422.

Kawano M, Kawasaki A, Sakata-Haga H, Fukui Y, Kawano H, Nogami H, Hisano S (2006) Particular subpopulations of midbrain and hypothalamic dopamine neurons express vesicular glutamate transporter 2 in the rat brain. J Comp Neurol 498:581-592.

Lavin A, Nogueira L, Lapish CC, Wightman RM, Phillips PE, Seamans JK (2005) Mesocortical dopamine neurons operate in distinct temporal domains using multimodal signaling. J Neurosci 25:5013-5023.

Liley AW (1956) An investigation of spontaneous activity at the neuromuscular junction of the rat. J Physiol 132:650-666.

Lodge DJ, Grace AA (2006) The laterodorsal tegmentum is essential for burst firing of ventral tegmental area dopamine neurons. Proc Natl Acad Sci U S A 103:5167-5172.

Manseau F, Danik M, Williams S (2005) A functional glutamatergic neurone network in the medial septum and diagonal band area. J Physiol 566:865-884.

Margolis EB, Hjelmstad GO, Bonci A, Fields HL (2005) Both kappa and mu opioid agonists inhibit glutamatergic input to ventral tegmental area neurons. J Neurophysiol 93:3086-3093.

Margolis EB, Lock H, Chefer VI, Shippenberg TS, Hjelmstad GO, Fields HL (2006) Kappa opioids selectively control dopaminergic neurons projecting to the prefrontal cortex. Proc Natl Acad Sci U S A 103:2938-2942.

Margolis EB, Fields HL, Hjelmstad GO, Mitchell JM (2008) Delta-opioid receptor expression in the ventral tegmental area protects against elevated alcohol consumption. J Neurosci 28:12672-12681.

Nair-Roberts RG, Chatelain-Badie SD, Benson E, White-Cooper H, Bolam JP, Ungless MA (2008) Stereological estimates of dopaminergic, GABAergic and glutamatergic neurons in the ventral tegmental area, substantia nigra and retrorubral field in the rat. Neuroscience 152: $1024-1031$.

Nitsch C, Riesenberg R (1988) Immunocytochemical demonstration of GABAergic synaptic connections in rat substantia nigra after different lesions of the striatonigral projection. Brain Res 461:127-142.

Omelchenko N, Sesack SR (2005) Laterodorsal tegmental projections to identified cell populations in the rat ventral tegmental area. J Comp Neurol 483:217-235.

Omelchenko N, Sesack SR (2007) Glutamate synaptic inputs to ventral teg- 
mental area neurons in the rat derive primarily from subcortical sources. Neuroscience 146:1259-1274.

Omelchenko N, Sesack SR (2009) Ultrastructural analysis of local collaterals of rat ventral tegmental area neurons: GABA phenotype and synapses onto dopamine and GABA cells. Synapse 63:895-906.

Overton P, Clark D (1992) Iontophoretically administered drugs acting at the N-methyl-D-aspartate receptor modulate burst firing in A9 dopamine neurons in the rat. Synapse 10:131-140.

Paladini CA, Iribe Y, Tepper JM (1999) GABAA receptor stimulation blocks NMDA-induced bursting of dopaminergic neurons in vitro by decreasing input resistnace. Brain Res 832:145-151.

Paxinos G, Watson C (2007) The rat brain in stereotaxic coordinates. Boston: Elsevier Academic.

Peters A, Palay SL, Webster Hd (1991) The fine structure of the nervous system: neurons and their supporting cells. New York: Oxford UP.

Reynolds ES (1963) The use of lead citrate at high $\mathrm{pH}$ as an electron-opaque stain in electron microscopy. J Cell Biol 17:208-212.

Ribak CE, Vaughn JE, Saito K, Barber R, Roberts E (1976) Immunocytochemical localization of glutamate decarboxylase in rat substantia nigra. Brain Res 116:287-298.

Sarabi A, Hoffer BJ, Olson L, Morales M (2001) GFRalpha-1 mRNA in dopaminergic and nondopaminergic neurons in the substantia nigra and ventral tegmental area. J Comp Neurol 441:106-117.

Schultz W (1998) Predictive reward signal of dopamine neurons. J Neurophysiol 80:1-27.

Schultz W (2002) Getting formal with dopamine and reward. Neuron 36:241-263.

Shepard JD, Chuang DT, Shaham Y, Morales M (2006) Effect of methamphetamine self-administration on tyrosine hydroxylase and dopamine transporter levels in mesolimbic and nigrostriatal dopamine pathways of the rat. Psychopharmacology (Berl) 185:505-513.

Sombers LA, Beyene M, Carelli RM, Wightman RM (2009) Synaptic overflow of dopamine in the nucleus accumbens arises from neuronal activity in the ventral tegmental area. J Neurosci 29:1735-1742.
Staley KJ, Mody I (1991) Integrity of perforant path fibers and the frequency of action potential independent excitatory and inhibitory synaptic events in dentate gyrus granule cells. Synapse 9:219-224.

Swanson LW (1982) The projections of the ventral tegmental area and adjacent regions: a combined fluorescent retrograde tracer and immunofluorescence study in the rat. Brain Res Bull 9:321-353.

Tagliaferro P, Morales M (2008) Synapses between corticotropin-releasing factor-containing axon terminals and dopaminergic neurons in the ventral tegmental area are predominantly glutamatergic. J Comp Neurol 506:616-626.

Takamori S, Rhee JS, Rosenmund C, Jahn R (2001) Identification of differentiation-associated brain-specific phosphate transporter as a second vesicular glutamate transporter (VGLUT2). J Neurosci 21:RC182.

Van Bockstaele EJ, Pickel VM (1995) GABA-containing neurons in the ventral tegmental area project to the nucleus accumbens in rat brain. Brain Res 682:215-221.

Varoqui H, Schäfer MK, Zhu H, Weihe E, Erickson JD (2002) Identification of the differentiation-associated $\mathrm{Na}+/ \mathrm{PI}$ transporter as a novel vesicular glutamate transporter expressed in a distinct set of glutamatergic synapses. J Neurosci 22:142-155.

Wise RA (2004) Dopamine, learning and motivation. Nat Rev Neurosci 5:483-494.

Xiao C, Zhou C, Li K, Davies DL, Ye JH (2008) Purinergic type 2 receptors at GABAergic synapses on ventral tegmental area dopamine neurons are targets for ethanol action. J Pharmacol Exp Ther 327:196-205.

Xiao C, Shao XM, Olive MF, Griffin WC $3^{\text {rd }}$, Li KY, Krnjeviæ K, Zhou C, Ye $\mathrm{JH}$ (2009) Ethanol facilitates glutamatergic transmission to dopamine neurons in the ventral tegmental area. Neuropsychopharmacology 34:307-318.

Xiao X, Li J, Samulski RJ (1998) Production of high-titer recombinant adeno-associated virus vectors in the absence of helper adenovirus. J Virol 72:2224-2232.

Yamaguchi T, Sheen W, Morales M (2007) Glutamatergic neurons are present in the rat ventral tegmental area. Eur J Neurosci 25:106-118. 